\title{
The Cold Mass Support System and the Helium Cooling System for the MICE Focusing Solenoid*
}

\author{
S. Q Yang, M. A. Green, W. W. Lau, R. S. Senanayake, B. Strauss, and H. Witte
}

\begin{abstract}
The heart of the absorber focus coil (AFC) module for the muon ionization cooling experiment (MICE) is the two-coil superconducting solenoid that surrounds the muon absorber. The superconducting magnet focuses the muons that are cooled using ionization cooling, in order to improve the efficiency of cooling. The coils of the magnet may either be run in the solenoid mode (both coils operate at the same polarity) or the gradient (the coils operate at opposite polarity). The AFC magnet cold mass support system is designed to carry a longitudinal force up to $700 \mathrm{kN}$. The AFC module will be cooled using three pulse tube coolers that produce $1.5 \mathrm{~W}$ of cooling at $4.2 \mathrm{~K}$. One of the coolers will be used to cool the liquid (hydrogen or helium) absorber used for ionization cooling. The other two coolers will cool the superconducting solenoid. This report will describe the MICE AFC magnet. The cold mass supports will be discussed. The reasons for using a pulsed tube cooler to cool this superconducting magnet will also be discussed.

Index Terms - Superconducting Solenoids, and Cooling
\end{abstract}

\section{INTRODUCTION}

$\mathrm{T}$ he development of a muon collider or a neutrino factory requires that beams of low emittance muons be produced. A key to the production of low emittance muons is muon ionization cooling. A demonstration of muon cooling is essential to the development of muon accelerators and storage rings [1]. The international Muon Ionization Cooling Experiment (MICE) will be a demonstration of muon cooling in a configuration of superconducting magnets [2] and absorbers that may be useful for a neutrino factory. Ionization cooling of muons occurs within an absorber that is located in the absorber focus coil module (AFC module). Ionization cooling occurs when there is a net loss of transverse muon momentum when the muons pass through the absorber material and are reaccelerated by adjacent RF cavities.

This report describes the AFC module two-coil focusing magnet that provides muon beam focusing within the absorber, where the ionization cooling occurs. This report discusses the focusing magnet cold mass support system and the cooling of the magnet with a pair of $1.5 \mathrm{~W}$ coolers.

Manuscript received 29 August 2006. M. A. Green (email: MAGreen(1bl.gov) is from the Lawrence Berkeley National Laboratory, Berkeley CA 94720 USA. S. Q. Yang, W. W. Lau, R. S. Senanayake, and H. Witte are from the Oxford University Physics Department, Oxford OX1-3RH, United Kingdom. B. Strauss is with the office of Science US Department of Energy, Germantown USA * This work was supported by the Office of Science, United States Department of Energy, under DOE contract DE-AC0205CH11231. DOE funding for the US Neutrino Factory and Muon Collider Collaboration is gratefully acknowledged.

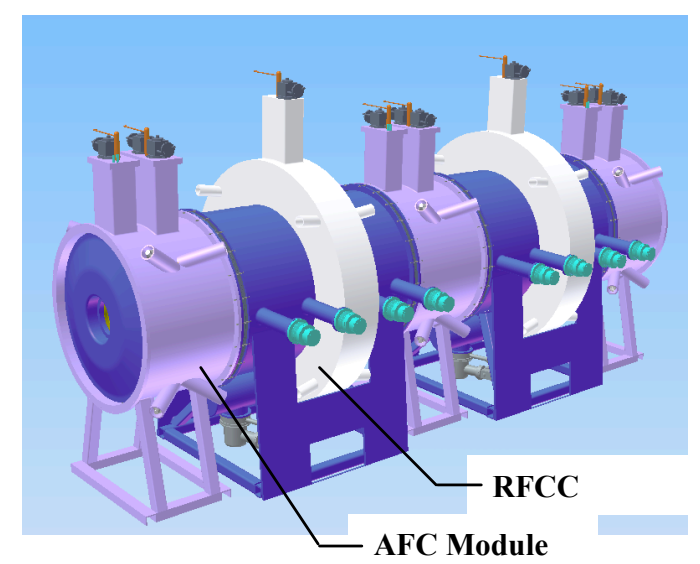

Fig. 1. A 3D View of the Central Section of the MICE Cooling Channel during the Final Stage of the Experiment.

\section{MICE AND THE AFC MODULE}

The proposed MICE experiment will test cooling on a low intensity muon beam from the ISIS ring at the Rutherford Appleton Laboratory in the United Kingdom. Once the muons have been produced and fed into the MICE channel at the desired emittance, the muon beam emittance is measured in the first tracker module [3]. From here the muon beam is fed into the center section of the cooling channel where cooling and reacceleration take place. The central cooling section of the MICE during the final stage of the experiment is shown in Fig. 1. Both longitudinal and transverse momenta are removed in the AFC module. The longitudinal momentum of the muons is replaced by RF acceleration in the RFCC module. The process of cooling and reacceleration occurs in the alternating AFC modules and RFCC modules. The beam emittance after cooling will be measured in the second tracker module at the end of the channel.

The three AFC modules are where the beam is focused and the muon momentum is removed by the absorber through ionization cooling. The AFC module shown in Fig. 1 consists of a superconducting magnet that surrounds the absorber that reduces the muon momentum in all directions. A three dimensional view of the AFC module is shown in Fig. 2. The AFC module will be cooled using three coolers that generate $1.5 \mathrm{~W}$ of cooling at $4.2 \mathrm{~K}$. One cooler will be used to cool the liquid absorber. The other two coolers will be used to cool the superconducting focusing magnet. A cross section of the AFC module showing the focusing magnet and a liquid absorber is shown in Fig. 3. The three coolers are not shown in Fig. 3. 


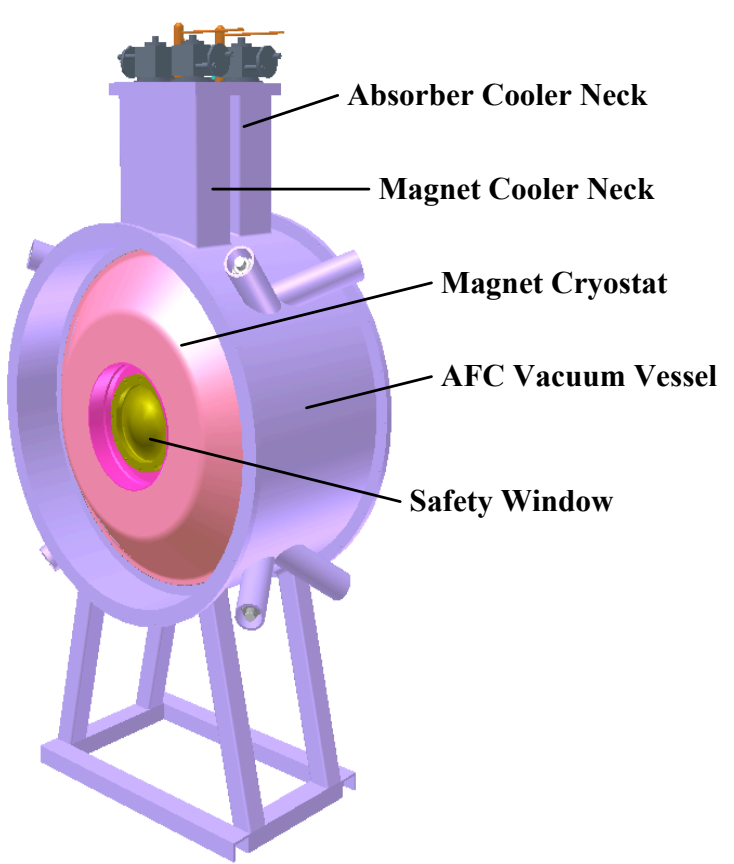

Fig. 2. A 3D View of the MICE AFC Module from the Magnet Cooler End of the Module

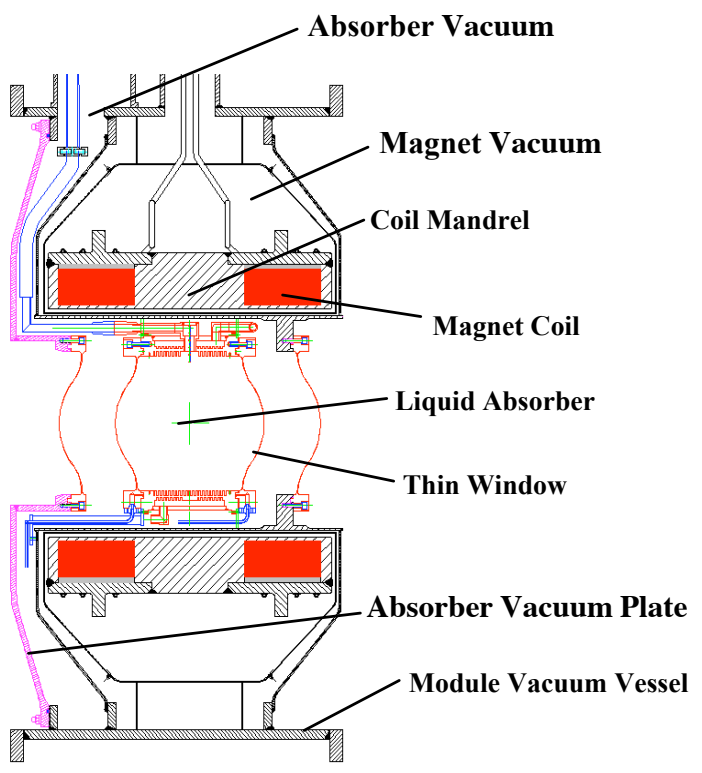

Fig. 3. A Cross-section of the AFC Module showing the Two-coil Superconducting Solenoid and the Absorber

The focusing magnet is designed to operate in both the gradient mode (flip mode) with the two coils operating at opposite polarity or the solenoid mode (non-flip mode) where the coils operate at the same polarity [4]. The forces and fields at the conductor are much higher when the focusing magnet operates in the flip mode [5]. Table 1 shows the parameters for the MICE focusing magnets while they operate in either mode at the highest muon momentum $(240 \mathrm{MeV} / \mathrm{c})$. From Table 1, it is clear that the flip mode is the worst mode in terms of peak field at the winding, temperature margin, and inter-coil magnetic forces. During a fault, the forces on the cold mass supports are also the higher.
Table 1. The Basic Operating Parameters of the MiCE Focusing MAGNET IN THE FLIP AND NON-FLIP MODES

\begin{tabular}{|c|c|c|}
\hline Parameter & Non-flip & Flip \\
\hline Coil Inner Radius (mm) & \multicolumn{2}{|c|}{263} \\
\hline Coil Thickness (mm) & \multicolumn{2}{|c|}{84} \\
\hline No. of Layers & \multicolumn{2}{|c|}{76} \\
\hline No. Turns per Layer & \multicolumn{2}{|c|}{127} \\
\hline Magnet $\mathrm{J}\left(\mathrm{A} \mathrm{mm} \mathrm{m}^{-2}\right)^{*}$ & 72.0 & 138.2 \\
\hline Magnet Current (A)* & 130.5 & 250.7 \\
\hline Magnet Self Inductance (H) & 137.4 & 98.6 \\
\hline Peak Induction in Coil $(\mathrm{T})^{*}$ & 5.04 & 7.67 \\
\hline Magnet Stored Energy (MJ)* & 1.17 & 3.10 \\
\hline $4.2 \mathrm{~K}$ Temp. Margin $(\mathrm{K})^{*}$ & $\sim 2.0$ & $\sim 0.6$ \\
\hline Inter-coil Z Force $(\mathrm{MN})^{*}$ & 0.56 & 3.53 \\
\hline
\end{tabular}

\section{FoCUSING MAGNET COLD MASS SUPPORT SYSTEM}

The proposed cold mass support system for the AFC magnet is self-centering [6]. A self-centering support system has the property that the physical center of the magnet does not change as the magnet is cooled from $300 \mathrm{~K}$ to $4 \mathrm{~K}$. The support system for any MICE magnet must withstand the forces put on the magnet during a quench or a fault as well as when the MICE channel is normally operated. During normal operation, the center AFC magnet cold mass support system has almost no longitudinal force on it. During normal operation, the end AFC modules may see a support system longitudinal force of up to $150 \mathrm{kN}$ (15 tons). The overall spring constant of the cold mass supports and external supports must be high enough so that the magnet center does not move more than $\pm 1 \mathrm{~mm}$ during normal operation and the angle of the axis of the magnet should not change more than $\pm 0.7 \mathrm{mrad}$ during normal operation. The design force for the AFC magnet cold mass support is determined by quench and other off normal operating conditions. The worst-case quench results in a 40-ton longitudinal force on the focusing magnet. The worst-case overall occurs when MICE operates in the flip mode with the current leads of one (or both coupling coils) reversed. This results in a cold mass support longitudinal force of 68 tons when the muon $p=240 \mathrm{MeV} / \mathrm{c}$ and $\square=420$ $\mathrm{mm}$. The AFC magnet cold mass support system design force is $700 \mathrm{kN}$ ( $\sim 70$ tons).

The self-centering cold mass support system proposed for the AFC magnet consists of eight supports strap assemblies (four at each end of the magnet). Each support strap assembly consists of two oriented fiberglass epoxy support bands with attachment hardware at each end and an intermediate temperature intercept between the two bands. The intermediate temperature intercept is tied to the first stage of the coolers that cool the AFC magnet. The intercept temperature is expected to be between 40 and $50 \mathrm{~K}$. The warm ends of the cold mass supports will be near the AFC vacuum vessel ends at azimuthal angles of $45,135,225$, and 315 degrees. The cold ends will be at the same angles but off by plus or minus 10 degrees (depending on the end) toward the mid-plane. A three dimensional view of the AFC cold mass support system is shown in Fig. 4. 


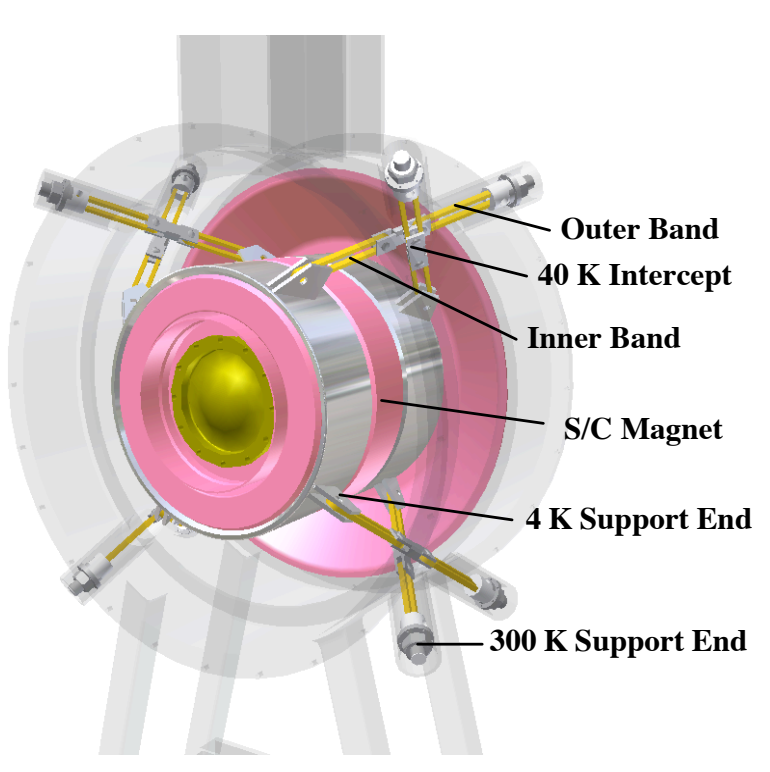

Fig. 4. A 3D View of the Focusing Magnet Cold Mass Supports

For the cold mass supports shown in Fig. 4 the design prestress will be $250 \mathrm{MPa}$ across the band, when the cold mass is at $4.2 \mathrm{~K}$. For the nominal design, the pre-stress at $300 \mathrm{~K}$ will be about $170 \mathrm{MPa}$. The nominal length for both the warm and the cold tension bands will be about $210 \mathrm{~mm}$. Each band will have two straps with a width of $25 \mathrm{~mm}$ and a thickness of 16.8 $\mathrm{mm}$. When the temperature at the intercept between the bands is $50 \mathrm{~K}$, the total heat leak to $4 \mathrm{~K}$ is about $0.11 \mathrm{~W}$. If the intercept temperature is raised to $77 \mathrm{~K}$ the heat leak into the magnet will be about $0.28 \mathrm{~W}$. The total heat flow into the intercepts will be about $2.7 \mathrm{~W}$. The final support system heat leak will be within a factor of two of the values given above.

\section{COOLING THE AFC MAGNET}

Each AFC magnet will be cooled using a pair of $1.5 \mathrm{~W}$ (at $4.2 \mathrm{~K}$ ) coolers. A single cooler will keep the liquid absorber cold (at $20 \mathrm{~K}$ for $\mathrm{LH}_{2}$ or $4.5 \mathrm{~K}$ for $\mathrm{LHe}$ ). The selection of the cooler is dependent on the magnetic field at the cooler. There are two types of coolers commercially available for cooling the magnets. They are the Gifford McMahon (GM) cooler and the pulse tube cooler. A model that generates $1.5 \mathrm{~W}$ at $4.2 \mathrm{~K}$ is commercially available for both types of coolers [7].

The critical cooler selection issue for MICE is the stray magnetic field outside of the magnets. The MICE channel doesn't have an iron shield to return the magnetic flux and shield the coolers. Both types of coolers have at least one component that is sensitive to magnetic fields of $0.05 \mathrm{~T}$ or more. An advantage of the pulse tube cooler is the fact that field sensitive part (the rotary valve drive motor) is further from the second stage cold head. The rotary valve motor can be shielded and it can be located remotely away from the cooler top plate, to a place where the magnetic field is lower.

The highest field at the cooler occurs when the magnet is in the flip mode at $p=240 \mathrm{MeV} / \mathrm{c}$ and $\square=420 \mathrm{~mm}$. This field is in the radial direction. The field magnitude is $\sim 0.6 \mathrm{~T}$ at second stage cold head and $\sim 0.35 \mathrm{~T}$ at a radius of the drive motor. When the AFC magnet is operated in the non-flip mode, the field at the sensitive parts of the cooler is much lower. The non-flip mode stay field is longitudinal.
TABle 2. Estimated AFC Magnet Heat LoAds AT $40 \mathrm{~K}$ AND $4.2 \mathrm{~K}$

Heat Leak

Component

(W)

\begin{tabular}{lcc} 
& $@ 40 \mathrm{~K}$ & $@$ @ $4 \mathrm{~K}$ \\
\hline Cold Mass Supports & $\sim 3$ & $\sim 0.22$ \\
Radiation through MLI & $\sim 6$ & $\sim 0.20$ \\
Necks and Instrumentation & $\sim 9$ & $\sim 0.50$ \\
Current Leads & $\sim 48$ & $\sim 0.80$ \\
Total Estimated Heat Leak & $\sim 66$ & $\sim 1.72$ \\
\hline
\end{tabular}

The projected heat loads for the AFC magnet are shown in Table 2. A pair $1.5 \mathrm{~W}$ pulse tube coolers appears to be an attractive choice for the AFC magnet. The heat loads shown in Table 2 suggest that the cooler first stage temperature of the pulse tube cooler will be less than $40 \mathrm{~K}$. The second stage temperature might be as low as $3.7 \mathrm{~K}$. Given the low operating temperature margin for the focusing magnet in the flip mode at $p=240 \mathrm{MeV} / \mathrm{c}$ and $\square=420 \mathrm{~mm}$, cooling the AFC magnet with a single cooler does not appear to be viable. The use of two coolers to cool the magnet can potentially increase the magnet temperature margin and it will allow the rotary valve for a pulse tube cooler to be in a low field region. Moving the rotary valve motor to a remote location will eliminate the need for a magnetic shield, thus reducing the field aberrations within the bore of the magnet.

Figure 5 shows the location of the magnet coolers and the absorber cooler as seen looking at the top of the AFC cryostat. The magnet cooler is within a He gas filled sleeve. Liquid helium leaves from the bottom of the helium sleeve. Figure 6 shows the two magnet coolers and the HTS and copper lead assemblies for the AFC magnet. The pipes that feed liquid helium to the bottom of the magnet are also shown in Fig. 6. The pipes that receive helium gas from the magnet and deliver the gas to the cooler sleeve is also shown.

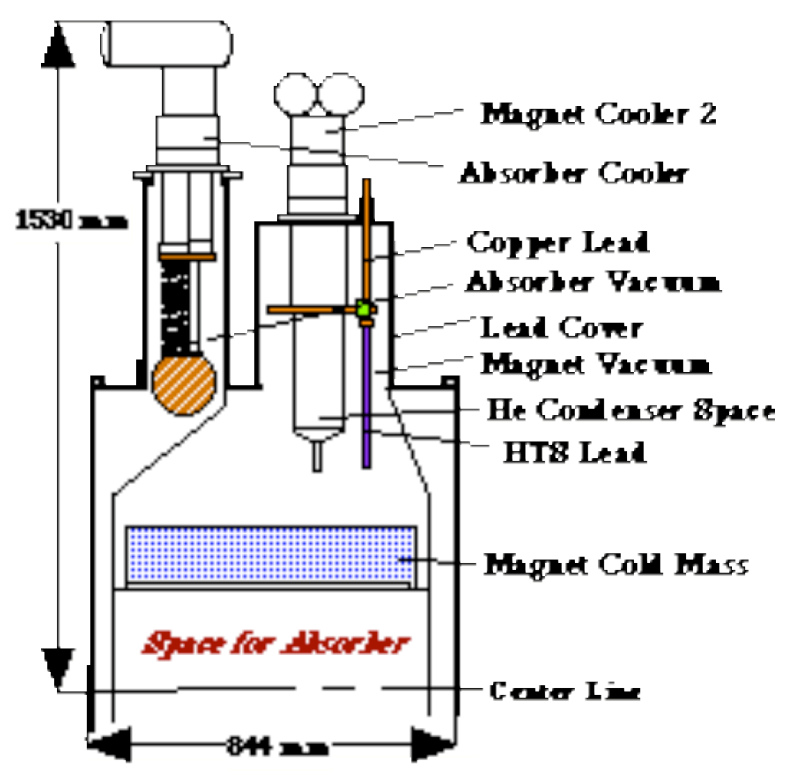

Fig. 5. AFC Cooler Location from the Side 


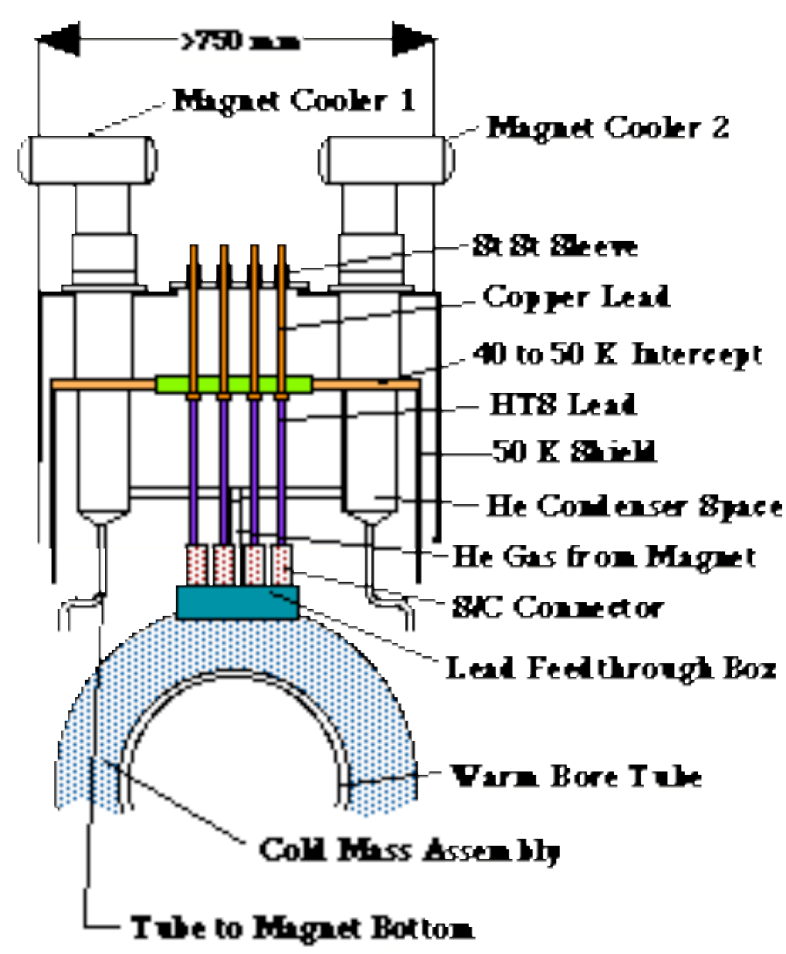

Fig. 6. The magnet Current Lead Assembly and the Two Magnet Coolers as seen from the Magnet End of the AFC Module

The bottom of the HTS leads is cooled by conduction through copper and $\mathrm{Nb}$ - Ti leads that feed directly from the liquid helium space. The HTS leads are assumed to be in vacuum. The upper part of the HTS leads is cooled directly by conduction from the first stage of the coolers. The first stage temperature is expected to be about $40 \mathrm{~K}$. The first stage removes the heat that comes down the copper current leads as well as heat leaks down the upper part of the cold mass supports, the MLI, instrumentation wires and the vent tubes.

The key to being able to operate the AFC magnet so that beams with a momentum of $240 \mathrm{MeV} / \mathrm{c}$ can be measured in MICE is reducing the temperature drop between the magnet hot spot and the cooler second stage cold heads [8]. The best ways of achieving a low temperature drop are; 1) reducing the heat flow into the $4 \mathrm{~K}$ region, 2) cooling the entire surface of the magnet instead of a single spot, and 3) using a thermal siphon heat pipe to connect the cooler second stage to the liquid helium that is around the magnet coils.

In order to achieve temperature drops of less than $0.1 \mathrm{~K}$ from the surface of the magnet in contact with liquid helium to the second stage of the cooler, the proper heat flow per unit area must occur at the condenser and on the magnet surface in contact with the helium. The heat flow per unit vertical surface of the condenser must be $<50 \mathrm{~W} \mathrm{~m}^{-2}$. For $1.5 \mathrm{~W}$, the condenser area must be $>0.03 \mathrm{~m}^{2}$. The heat flow from the exposed magnet surface to the liquid helium around the magnet must be $\sim 3 \mathrm{~W} \mathrm{~m}^{-2}$ for a $\square \mathrm{T}$ at the surface of $0.03 \mathrm{~K}$. This suggests that the total magnet surface exposed to liquid helium must have an area of about $\sim 0.65 \mathrm{~m}^{2}$.

\section{CONCLuding COMMENTS}

The AFC contains a superconducting magnet that focuses the muon beam to low values of beam beta, in order to improve ionization cooling efficiency. The two coil superconducting solenoid is design to be operated in either the gradient mode (the flip mode) or the straight solenoid mode (the non-flip mode). When the magnet is operated in the flip mode the fields and forces are generally higher than when the magnet operates in the non-flip mode. When MICE operates with muons at an average momentum of $240 \mathrm{MeV} / \mathrm{c}$, the focusing magnet temperature margin is about $0.6 \mathrm{~K}$ when the magnet operates at $4.2 \mathrm{~K}$.

It is expected that the AFC magnet cold mass will be supported by a self-centering tension band support system capable of carrying $700 \mathrm{kN}$ ( $\sim 70$ tons) in the longitudinal direction and at least $50 \mathrm{kN}$ ( $\sim 5$ tons) in the radial direction. The cold mass support system will have a thermal intercept at about $40 \mathrm{~K}$. Cooling for this intercept will come from the first stage of the coolers.

The AFC solenoid can be cooled using two coolers. The coolers will provide cooling for the copper current leads, the cold mass supports, other conductive heat loads and the radiation heat load through the MLI. The first stage of the cooler is expected to operate at about $40 \mathrm{~K}$. The cooler second stage intercepts all heat leaks into the cold mass as well as heat leaks down the HTS leads that connect to the copper current leads that come in from $300 \mathrm{~K}$. The cooler second stage will be connected to magnet cold mass using a pair of thermal siphon heat pipes. The AFC module will have a third $4 \mathrm{~K}$ cooler to cool the liquid absorber.

\section{REFERENCES6)}

[1] R. B. Palmer, A. Sessler, A. Skrinsky, A. Tollestrup, et al, "Muon Colliders, " Brookhaven National Laboratory Report BNL-62740, January 1996

[2] G. Gregoire, G. Ryckewaert, L. Chevalier, et al, "MICE and International Muon Ionization Cooling Experiment Technical Reference Document," http://hep04.phys.itt.edu/cooldemo

[3] M. A. Green, C. Y. Chen, T. Juang et al, "The Design Parameters for the MICE tracker Solenoid," IEEE Transactions on Applied Superconductivity 17, No. 1, (This volume), (2007).

[4] S. Q. Yang, M. A. Green, et al, "The Mechanical and Thermal Design for the MICE Focusing Solenoid Magnet System," IEEE Transactions on Applied Superconductivity 15, No. 2, p 1259, (2005).

[5] M. A. Green, Y. Ivanyushenkov, W. W. Lau et al, "Progress on the Focus Coils for the MICE Channel," Proceedings of the 2005 Particle Accelerator Conference, p 3417 http://hep04.phys.itt.edu/cooldemo, MICE Note 118 (2005)

[6] M. A. Green, R. S. Senanayake, "The Cold Mass Support System for the MICE Focusing and Coupling Magnets," MICE Note 106, (2004), http://hep04.phys.itt.edu/cooldemo

[7] M. A. Green, "Cooling the MICE Magnets using Small Cryogenic Coolers," http://hep04.phys.itt.edu/cooldemo, MICE Note 109, (2004)

[8] M. A. Green, "How the Performance of a Superconducting Magnet is affected by the Connection between a Small Cooler and the Magnet," IEEE Transactions on Applied Superconductivity 16, No. 1, p 1330, (2006) 
DISCLAIMER

This document was prepared as an account of work sponsored by the United States Government. While this document is believed to contain correct information, neither the United States Government nor any agency thereof, nor The Regents of the University of California, nor any of their employees, makes any warranty, express or implied, or assumes any legal responsibility for the accuracy, completeness, or usefulness of any information, apparatus, product, or process disclosed, or represents that its use would not infringe privately owned rights. Reference herein to any specific commercial product, process, or service by its trade name, trademark, manufacturer, or otherwise, does not necessarily constitute or imply its endorsement, recommendation, or favoring by the United States Government or any agency thereof, or The Regents of the University of California. The views and opinions of authors expressed herein do not necessarily state or reflect those of the United States Government or any agency thereof, or The Regents of the University of California. 


\title{
The Cold Mass Support System and the Helium Cooling System for the MICE Focusing Solenoid*
}

\author{
S. Q Yang ${ }^{a}$, M. A. Green ${ }^{b}$ W. W. Lau ${ }^{a}$, R. S. Senanayake ${ }^{a}$, \\ B. Strauss ${ }^{c}$, and H. Witte ${ }^{a}$
}

a) Oxford University Physics Department, Oxford OX1-3RH, UK b) Lawrence Berkeley Laboratory, Berkeley CA 94720, USA c) Office of Science US Department of Energy, Germantown MD, USA

*This work was supported by the Office of Science, United States Department of Energy, under DOE contract DE-AC02-05CH11231. DOE funding for the US Neutrino Factory and Muon Collider Collaboration is gratefully acknowledged. 